\title{
2010 CAEP/ACMU Scientific Abstracts Author Index
}

\section{$\underline{\text { AUTHOR }}$}

A

Abu-Laban RB

Ackroyd-Stolarz S

Afilalo M

Agarwal D

Ali S

Al-Qadhi S

Alrajhi K

Andolfatto $\mathrm{G}$

Ang L

Angelski CL

Anstett D

Anthony CJ

Archambault P

Arcieri V

Arntfield R

Aubin KA

\section{B}

Babul S

Ballantine M

Bandiera GW

Barbic D

Barbic S

Barrowman N

Baw B

Bawden J

Bawden JL

Beatty L

Beland C

Bellazzini MA

Belleville G

Berger PE

Bergeron É

Bhanji $\mathrm{F}$

Bhatt M

Bhimani M

Birnie D

Black K

Black KJL

Blakney G

Blanchet $M$

Blouin D

Bonacci A

Bond $\mathrm{C}$

Bond K

\begin{tabular}{|c|c|}
\hline \multirow[t]{4}{*}{ ABSTRACT No. } & Borgundvaag B \\
\hline & Boychuk B \\
\hline & Boyko D \\
\hline & Brehaut J \\
\hline 14,73 & Brison RJ \\
\hline 147 & Brooks S \\
\hline 50 & Brophy J \\
\hline 40,44 & Brown A \\
\hline 86,96 & Brubacher JR \\
\hline 160 & Bruder E \\
\hline 1,84 & Bullard M \\
\hline $19,36,45$ & \\
\hline 108 & Bullard MJ \\
\hline 72 & \\
\hline $100,121,124$ & C \\
\hline 147 & Calder L \\
\hline 163,164 & Camargo CA \\
\hline 3 & Campbell RL \\
\hline 85 & Campbell S \\
\hline 158 & Cappelli M \\
\hline & Carter A \\
\hline & Cembrowski G \\
\hline 133 & Chabot P \\
\hline 162 & Chahal AM \\
\hline 117 & Chan H \\
\hline 6 & Chan MC \\
\hline 6 & Chandra S \\
\hline 118 & Channan $\mathrm{P}$ \\
\hline 143 & Charette C \\
\hline $20,100,121,124$ & Charette $M$ \\
\hline 140 & Chauny J \\
\hline 135 & Chauny JM \\
\hline 70 & Cheng AL \\
\hline 76,109 & Chipman ML \\
\hline 119 & Chisholm D \\
\hline 163 & Cho D \\
\hline 131 & Choi S \\
\hline 103,112 & Christenson J \\
\hline 68 & Christie R \\
\hline 154,162 & Church K \\
\hline $22,46,56$ & Ciampi A \\
\hline 68 & Clark E \\
\hline 72 & Clark K \\
\hline 115 & Clayden R \\
\hline 158 & Clement CM \\
\hline 81 & Colacone A \\
\hline 165 & Constas $\mathrm{N}$ \\
\hline 106 & Coome GE \\
\hline 140 & Corrin $M$ \\
\hline
\end{tabular}

\begin{tabular}{|c|c|}
\hline $22,46,56$ & Cripton PA \\
\hline 93 & Crooks J \\
\hline $100,121,124,140$ & Croskerry C \\
\hline 9,15 & Crowder K \\
\hline $22,46,56$ & Curran J \\
\hline 58 & Curtis Lee A \\
\hline 136 & Curtis SJ \\
\hline $110,139,148$ & Cusimano MD \\
\hline $14,105,133,144$ & Cwinn AA \\
\hline 7 & Cyr-Hansen M \\
\hline $\begin{array}{r}4,17,32,41 \\
64,65,106,128\end{array}$ & j \\
\hline $10,20,24,62,115$ & D'Souza P \\
\hline & Dagnone JD \\
\hline & Dalgleish D \\
\hline 55 & Dallaire CD \\
\hline 61 & Dance E \\
\hline $53,99,127$ & Daoust R \\
\hline 31 & de Grave W \\
\hline 86 & Decker WW \\
\hline 63,101 & deJong $\mathrm{D}$ \\
\hline 115 & Delaney JS \\
\hline 33 & Demers ME \\
\hline 73 & Denny CJ \\
\hline 144 & Diner B \\
\hline 115 & DiodatiJG \\
\hline 40,44 & Dionne C \\
\hline 52 & Djogovic D \\
\hline 75 & Doan Q \\
\hline 9,15 & Dodd T \\
\hline 54,119 & Dong KA \\
\hline 136 & Dong SL \\
\hline 116 & Dowlatshahi D \\
\hline 133 & Dowling S \\
\hline 133 & \\
\hline 68 & Doyle-Waters MM \\
\hline 13,60 & Dreyer J \\
\hline $19,36,45,93$ & Dreyer JF \\
\hline 147 & Dubrovsky AS \\
\hline 120 & \\
\hline 82 & $\mathbf{E}$ \\
\hline 13 & Eby D \\
\hline 98,102 & Edmonds ML \\
\hline 42,92 & Elder B \\
\hline $22,28,46,47,56$ & Eltorki M \\
\hline 50 & Émond $M$ \\
\hline 160 & \\
\hline 138 & Erwin PJ \\
\hline 160 & Espinola JA \\
\hline
\end{tabular}

133

$100,121,124$

21

94

86

150

96

133

150

165

3

30

161

158

81

33

103

$53,99,127$

$19,36,45$

69

137,145

117

25

54, 119

33

106

68

3

81

24

43,49

$8,23,59,80$,

$91,108,123,130$

2, 77

85

27,74

3

126

13

68

$33,49,122,131$,

136, 137, 145, 153

44

61 
Evans J

Ezekowitz JA

F

Farion KJ

Fernandes CMB

Filiatrault L

FitzGerald JM

Fleet R

Flinn A

Foldes-Busque G

Forster A

Fortin C

Foster B

Frank JR

Fratu R

Fraulin F

Freedman S

Frendo T

Friedman SM

\section{G}

Gaebert D

Gagnon M

Gagnon MP

Gatien M

Geurts J

Godfrey C

Gokiert R

Goldman RD

Gottesman R

Gouin S

Grafstein E

Graham TAD

Green J

Green R

Greenberg G

Greenfield G

Grimshaw J

Guimont C

Guo XY

Gursky L

Guttmann A

\section{H}

Hagan JB

Hagel BE

Hall AK

Hall C

Hames H

Hamm MP

Harder C

Harriman D

Harris D

Harris MA

Harrop R

$$
\text { 100, 121, } 124
$$

115

35,161

$34,71,111$

73

73

54, 119, 156

147

54, 119

55,84

26

29

$13,107,150$

122

108

68

133

133

$100,121,124$

122

136

155

151

80

86

104

103

26, 68

$12,19,36,45$

$78,88,93,116,129$

115

123

135

13

23,91

$9,15,146$

68

4, 17, 32,

$41,64,65,128$

130

$16,95,141$

Islam R

Jackson P

Jarvis C

Jayaraman D

Jednak R

Jensen JL

Jewell J

Jhangri $G$

Johns C

Johnson D

Johnson DW

Joubert G

K

Kanji S

Kanthala AR

Kapur A

Karim S

Karkhaneh M

Kasaboski A

Kelly AM

99, 127 Kelly-Smith CH

Kerr B

Kim K

Kiss A

Klassen TP

Koonar H

Kozan DC

Krause J

Krishnan JA

Kulasegaram M

Kuramoto L

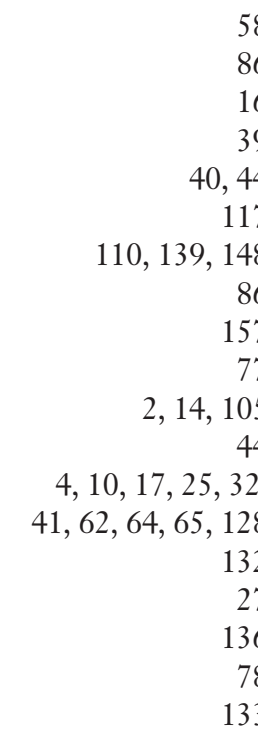

$4,8,12,17,19,22,23$,
$32,36,37,38,41,45$,
$56,57,64,65,78,80$
$88,91,93,128,129,130$

9
131

31,114

6

29

$15,18,21$,

$63,67,101$

$34,71,111$

132

13

68

96,146

68

135

$53,99,127$

13

147

125

$9,15,18$

47

2,77

130

152

3,117

146

8

115

$34,71,111$

61

52

14
L

Lalani M

Lali P

100,121

Lam NT

Lam TV

Lang E

Lang ES

Lange L

Langhan T

Langhan TS

Lapointe J

Latoszek K

Lavoie A

Le Sage N

Leaver CA

Lee CA

Lee D

Lehnhardt K

LeMay S

Leroux Y

Lessard M

Li JB

Li JT

Lim C

Lim R

Lin D

Lin S

Loewen PS

Logue EP

Loreto C

Lortie G

Louis $M$

M

MacKelvie J

MacLeod T

35,161

Magee K

Main C

Maire S

Marchand A

Mashinter LD

Mason S

McCusker J

McGahern C

McGillivray D

McGraw R

McKechnie K

McKnight RD

McLeod S

McLeod SL

35,161

$22,46,56,135$

87

166

54, 119

132

47

82

146

29

113

$34,71,110$,

$111,120,139,148$

$48,74,85$,

97, 126, 138, 142

McPhalen D

108

12,88

McPhee J

120

McVey J

Medrano OF 
Mendelsohn D

Mercier É

Mercuur L

Messier A

Meurer D

Meurer DP

Meyers C

Millar K

Millard W

Milne K

Milner RA

Minor KD

Montgomery C

Montori VM

Moola S

Mooney E

Moore K

Moore L

Morin J

Morin N

Morrison LJ

Mosdossy G

Mott D

Murad MH

Murphy N

Myers RP

\section{$\mathbf{N}$}

Ness R

Nestler D

Newton AS

Nolin P

Nosyk B

Novena A

Nowacki A

Nussbaum C

\section{O}

Oczkowski W

Ogilvie KS

O'Grady J

Osmond $\mathrm{MH}$

Ospina $M$

Ouellet MC

P

Palatnick W

Patocka C

Patrice J

Patterson $\mathrm{H}$

Pauls M

Payrastre J

Peddle $M$

Pelland M

Perry JJ

Peter S
$110,139,148$

153

$8,38,80$

26

106

$10,20,62$

69

108

48

162

104

76,109

$100,121,124,149$

44

156

3

7

$33,131,137,145,153$

33

26

3,58

120

31

44

147

66,79

156

40

86,96

131

14

142

152

13

5,11

60

164

$9,15,86$

$4,17,32$,

$41,64,65,128$

33

151

69

35

42, 92

51

42,92

85,120

54,119

22, 33, 43, 46,

$49,55,56,84,134$

14
Pickett W

Pilkington $M$

Pirie J

Piteau SJ

Plant J

Plint A

Plint AC

Poirier S

Poitras J

Porter R

Poulin C

Poureslami I

Preiksaitis J

Price I

Prowd C

R

Rang L

Reichl R

Reynolds CCO

Rizg K

Robert MA

Robert S

Rocca N

Rogenstein C

Rosychuk RJ

Roth VR

Rowe $\mathrm{BH}$

4, 10, 16, 17, 20, 22, 24, 25, $32,41,46,56,61,62,64,65$, $81,95,100,106,115,121,124$,

$125,128,132,140,141,149$

$\mathbf{S}$

Sahay S

Sales A

Salkeld E

Sanaee L

Saunders LD

Scheuermeyer F

Schneider S

Schull MJ

Schultz SE

Schultzer M

Schulzer M

Scott J

Scott S

Sedran R

Shah A

Shaheen AAM

Sharma M

Shaw MF

Shefrin AE

Sheps S

Sherbino J

Singer JS

Singh $M$

118,146

35,161

$54,119,158,163,164,166$

$12,78,88$

132

110, 139, 148

$34,71,111,120$

66,79

43,49

138

104
Sirois $M$

33

137,145

49

97

118 Skoretz T

156 Smallfield A

68 Smith B

Smith V

So J

Sobolev B

Sookram J

Soucy N

Spence G

Srigley JA

Stachura M

Stang AS

Steinert Y

Stempien J

Stenstrom R

Stephens D

Stewart J

Stiell IG

Stotts G

Strome T

Strumpf E

Stukel T

Sutherland J

Svenson JE

Sweet DD

Symington C

$110,139,148,154$ $34,71,111$ 40 58 14 149 50 130 87

$12,19,22,45$, $46,56,78,88,116$

\section{8}

112

$9,13,15,22,28,43,44$, $46,47,49,55,56,59,134$

43,49

151

82

90

43,49

76,109

2, 77

43,49

$\mathbf{T}$

Taljaard M 146

Tang PHP 87

Tanguay A 166

Taylor B 72

Taylor K 8

Terrett L 51

Teschke K 133

Thind A 27

Thiruganasambandamoorthy V 134

Titus C

Tran C

Travers AH

159

21,67

Tse $\mathrm{S}$

35

Tselios C

50

134

Turko E

15,18

U-V

Unger B

50

Upadhye S

$42,92,143$

Vadeboncoeur A

Vaidyanathan L

Vaillancourt C

Vaillancourt S

$1,9,13,15,55,75$

90 
Vaillancourt $\mathrm{V}$

van Wylick R

Vandermeer B

Vanier L

Verbeek PR

Verdon J

Vermeulen MJ

Verreault R

Vester M

Villa-Roel C

$4,10,17,20,24,32$

$41,62,64,65,100,106$,

$115,121,124,128,140,149$

Vilneff R

Voaklander DC

Voskamp D

W

Waite T
130

18

68

4, 17, 32, 41, $64,65,96,128$

136

3

16,95

33

$10,24,62$

\section{5}

161

Willis $\mathrm{V}$

3 Winters $M$
Winer LR

Wing A

Walker B

Walker M

Wall B

Wang D

Wasserman J

Weitzman B

Weldon E

Wells GA

elsford $M$

elsh $\mathrm{R}$

Wilkinson D

Willcockson I

Williams J

$\begin{array}{rl}130 & \text { Wong L } \\ 63,67,101 & \text { Woo MY } \\ 7 & \text { Woods R } \\ 8,23,38,80,91 & \text { Worster A } \\ 118 & \text { Worthington JR } \\ 43,49 & \text { Wright M } \\ 13 & \\ 151 & \mathbf{X}-\mathbf{Z} \\ 9,15,22,46,56,59 & \text { Xue X } \\ 3,52 & \text { Yarema MC } \\ 149 & \text { Yeung M } \\ 13 & \text { Yeung TC } \\ 114 & \text { Young B } \\ 123 & \text { Yoxon H } \\ 81 & \text { Yu E } \\ 124 & \text { Zaric GS } \\ 103 & \text { Zed PJ } \\ 124 & \text { Zhan C } \\ 133 & \end{array}$

4, 17, 32, 41, 64, 65, 128

$1,70,107,155$

94

$42,49,83,87,92$

70

35,161

$22,50,56$

$66,79,81$

13,150

2, 77

123

35,161

93

27

$14,135,147$

3, 58 